\title{
High Time-Resolved Cardiac Functional Imaging Using Temporal Regularization for Small Animal on a Clinical 3T Scanner
}

\author{
Bénédicte M. A. Delattre, Dimitri Van De Ville, Vincent Braunersreuther, Corinne Pellieux, Jean-Noël Hyacinthe, \\ René Lerch, François Mach, and Jean-Paul Vallée*
}

\begin{abstract}
Accurate assessment of mice cardiac function with magnetic resonance imaging is essential for longitudinal studies and for drug development related to cardiovascular diseases. Whereas dedicated small animal MR scanners are not readily available, it would be a great advantage to be able to perform cardiac assessment on clinical systems, in particular, in the context of translational research. However, mouse imaging remains challenging since it requires both high spatial and temporal resolutions, while gradient performances of clinical scanners often limit the reachable parameters. In this study, we propose a new cine sequence, named "interleaved cine," which combines two repetitions of a standard cine sequence shifted in time in order to reach resolution parameters compatible with mice imaging. More precisely, this sequence allows temporal resolution to be reduced to $6.8 \mathrm{~ms}$ instead of $\mathbf{1 3 . 5} \mathrm{ms}$ initially imposed by the system's hardware. We also propose a two-step denoising algorithm to suppress some artifacts inherent to the new interleaved cine thus allowing an efficient enhancement of the image quality. In particular, we model and suppress the periodic intensity pattern and further denoise the sequence by soft thresholding of the temporal Fourier coefficients. This sequence was successfully validated with mass and function measurements on relevant mice models of cardiovascular diseases.
\end{abstract}

Index Terms-Cine, denoising, heart function, I1-regularization, magnetic resonance imaging (MRI), mouse, 3T.

Manuscript received June 15, 2011; revised August 29, 2011 and October 14, 2011; accepted October 23, 2011. Date of publication November 1, 2011; date of current version March 21, 2012. This work was supported in part by the Swiss National Science Foundation under Grant PPOOB3-116901 and Grant PP00P2-123438 and in part by the Center for Biomedical Imaging of the Geneva and Lausanne Universities, EPFL, and the Leenaards and Louis-Jeantet foundations. Asterisk indicates corresponding author.

B. M. A. Delattre and J.-N. Hyacinthe are with the Division of Radiology, Faculty of Medicine, Foundation for Medical Researchers, Geneva University Hospital, University of Geneva, Geneva 1211, Switzerland (e-mail: benedicte.delattre@gmail.com; Jean-Noel.Hyacinthe@unige.ch).

D. Van De Ville is with the Institute of Bioengineering, Ecole Polytechnique Fédérale de Lausanne, Lausanne 1015, Switzerland, and also with the Department of Radiology and Medical Informatics, University of Geneva, Geneva 1211, Switzerland (e-mail: dimitri.vandeville@epfl.ch).

V. Braunersreuther, C. Pellieux, R. Lerch, and F. Mach are with the Division of Cardiology, Foundation for Medical Researchers, Department of Internal Medicine, University Hospital, Geneva 1211, Switzerland (e-mail: Vincent.Braunersreuther@unige.ch; corinne.Pellieux@unil.ch; rene. lerch@hcuge.ch; francois.Mach@hcuge.ch).

*J.-P. Vallée is with the Division of Radiology, Geneva University Hospital, University of Geneva, Faculty of Medicine, Foundation for Medical Researchers, Geneva 1211, Switzerland (e-mail: jean-paul.vallee@unige.ch).

Digital Object Identifier 10.1109/TBME.2011.2174363

\section{INTRODUCTION}

$\mathbf{M}$ AGNETIC resonance imaging (MRI) is a key tool to assess noninvasively and accurately cardiac function [1]. Despite the lower temporal resolution compared to echography, MRI has the advantage of more reproducible results and the ability to assess whole heart volume. With robust estimation of myocardial contraction, MRI is considered as the gold standard for cardiac function assessment [2], [3].

In a context where cardiovascular diseases are still the leading cause of mortality worldwide [4], small animal cardiac MR imaging is an important research topic for biology and medicine, notably with the development of knockout and transgenic (TG) mice to study the genes and mechanisms involved in cardiovascular diseases [5]. While most of the mouse studies have been performed on dedicated small animal MRI, these scanners are not available on all research sites. In this case, being able to run sequences to evaluate cardiac function of rodents on clinical scanners could be advantageous, especially for translational research.

Nevertheless, mouse imaging on clinical scanners remains challenging because of the small dimensions of the heart and the high heart-beating rate. Therefore, it requires some adjustments of the existing sequences [6]. Specifically, the tradeoff between sufficient signal-to-noise ratio (SNR) and adequate temporal and spatial resolution is high and reachable values are ultimately limited by gradient hardware. Following Roussakis et al. [7], 11 phases per cardiac cycle are at least necessary to evaluate ejection fraction (EF). Considering a mouse heart rate of 600 beats/min, the required time resolution should be at least $9 \mathrm{~ms}$ whereas, in dedicated scanners, the cardiac function is accurately estimated with a temporal resolution of $8.4 \mathrm{~ms}$ [8]; on most clinical scanners, the available cine sequence provides a temporal resolution in the range of 11-18 ms [9], [10]. There is thus a need to acquire more cardiac phases to assess accurately cardiac function. An alternative could be the use of a dedicated gradient insert to increase hardware capabilities, but this option is not always viable.

In this study, we propose a modification of the acquisition scheme through a new cine sequence that can reach a temporal resolution compatible with small animal imaging requirements; i.e., down to $6.8 \mathrm{~ms}$. This goal was achieved by combining two repetitions of the sequence where the second repetition is shifted in time in order to acquire additional data and validated using relevant mice models of cardiovascular diseases. 


\section{METHODS}

\section{A. Animal}

Two types of mice were used for this study, a group of male C57/BL6 wild type (WT) mice aged between 8 and 12 weeks $(n=7)$ as well as a group of female TG1306/R1 TG mice aged of 24 weeks $(n=14)$ as well as their WT littermates $(n=9)$. This last group has a C57/BL6 background and is known to develop a left ventricle hypertrophy [11], [12]. For the function measurements, a part of C57/BL6 WT mice underwent a complete ligation of left descendant coronary artery to produce a myocardial infarction (MI) and were scanned $24 \mathrm{~h}$ after the surgery $(n=3)$. This protocol was performed in agreement with the animal care committee.

\section{B. Sequence Design}

Images were acquired on a clinical 3T MR scanner (Magnetom TIM Trio, Siemens Medical Solutions, Erlangen, Germany) with a dedicated two-channel mouse receiver coil (Rapid biomedical GmbH, Rimpar, Germany). The basic sequence was a segmented turboflash cine sequence (already validated in other studies with slightly different parameters [9], [13]) with following parameters: FOV $111 \mathrm{~mm}$, in-plane resolution $257 \mu \mathrm{m}$, slice thickness $1 \mathrm{~mm}$, TE/TR $6.2 / 13.5 \mathrm{~ms}$, flip angle $30^{\circ}$, GRAPPA with acceleration factor 2 , two averages, and typical acquisition time per slice $3 \mathrm{~min}$. The sequence was respiratory and ECG gated. For each R-R cycle, only one line of $k$-space was acquired in order to minimize the repetition time $(T R)$. With this configuration, time resolution was ultimately limited by gradient hardware (maximum available amplitude and slew rate) to $T R=13.5 \mathrm{~ms}$. One obtains image series composed of $N$ phases separated by the time interval $T R$

$$
I\left(t_{a}\right)=m_{a}(x, y) \text { for } t_{a}=0, T R, 2 \cdot T R, \ldots, N \cdot T R
$$

where $I$ is the image series and $m$ is the measured image data along time for a given voxel. The proposed sequence (referred to as "interleaved cine") is composed of the repetition of the basic sequence where the second repetition is shifted in a time delay corresponding to half of the basic sequence's $T R$ (i.e., $6.8 \mathrm{~ms}$ ). In this case, the second image series is

$I\left(t_{b}\right)=m_{b}(x, y)$ for $t_{b}=0, \frac{T R}{2}, 3 \cdot \frac{T R}{2}, \ldots,(2 N+1) \cdot \frac{T R}{2}$.

Finally, the combination of the two repetitions $I\left(t_{a}\right)$ and $I\left(t_{b}\right)$ gives a cine with a time resolution of $6.8 \mathrm{~ms}(T R / 2)$ instead of $13.5 \mathrm{~ms}(T R)$ :

$$
\begin{aligned}
& I\left(t_{c}\right)=m_{c}(x, y) \\
& \text { for } t_{c}=0, \frac{T R}{2}, T R, 3 \cdot \frac{T R}{2}, 2 \cdot T R, \ldots,(2 N+1) \cdot \frac{T R}{2} .
\end{aligned}
$$

In Fig. 1, we show schematically the design of the basic cine as well as of the interleaved cine and their respective reconstruction. This scheme has the main advantage of keeping the total acquisition time constant between the two sequences.
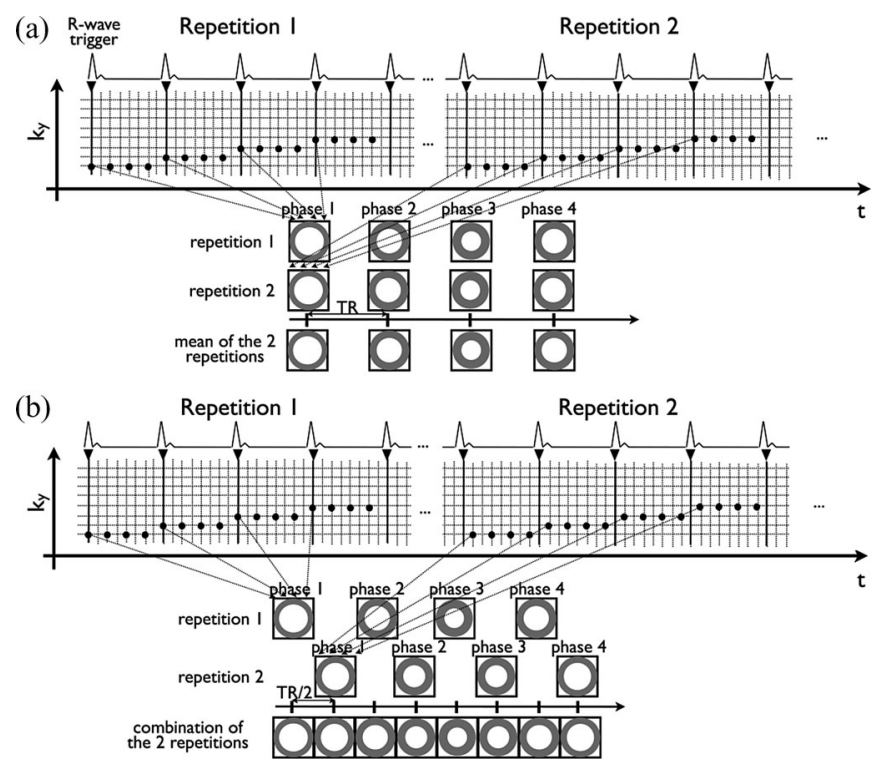

Fig. 1. Schematic representation of $k$ - $t$ space sampling and reconstruction designs (for clarity, we represented an example where only four cardiac phases are sampled in each repetition). (a) Basic cine and (b) interleaved cine, while the two repetitions of basic cine are averaged to form the final image series, the second repetition of the interleaved cine is shifted of $1 / 2 T R$, and the two combined repetitions form a series with double temporal resolution.

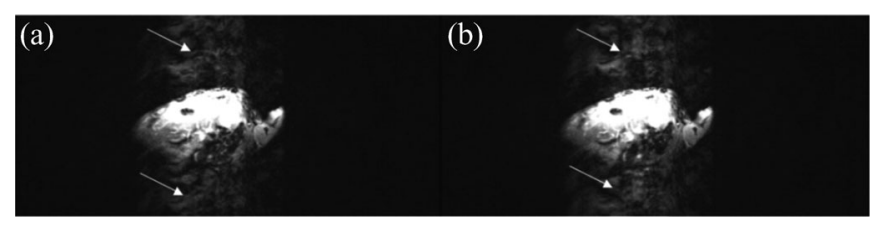

Fig. 2. Illustration of the ghosting artifacts that lead to the flickering artifact in the interleaved cine. Images are the first frame of cine repetitions acquired at the same location. Windowing was forced on purpose in order to have a better visualization of the artifacts. (a) Cine acquired with no trigger delay, and (b) shifted cine, acquired after a trigger delay of $1 / 2 T R$. (a) and (b) are the two cine composing the interleaved cine, white arrows are pointing areas where signal intensity of ghosting artifact is different between the two cine sequences.

\section{Image Enhancement}

The final combination of images in the interleaved cine has a lower SNR compared to the basic sequence since the two repetitions of images are no longer averaged, as it was the case in the basic sequence. Moreover, since the interleaved cine repetitions are acquired at different phases of the cardiac cycle, the flow artifacts are slightly different between the two repetitions as illustrated in Fig. 2. While repetition brings in new temporal information, the flow artifact resulted in a flickering effect when the two shifted cine repetitions were combined. In order to perform denoising and correction of the flickering effect, we defined the following image model $f_{c}$ for a measured value:

$$
f_{t}(x, y)=s_{t}(x, y)+A(x, y) \sin (\omega t+\varphi)+n_{t}(x, y)
$$

where $t$ is the temporal index, $(x, y)$ the spatial ones, $s$ represents the nondegraded signal, $A(x, y) \sin (\omega t+\phi)$ represents the flicker, and $n$ are the noise contributions. Noise was reduced by taking advantage of the periodicity of the cardiac cycle; i.e., we applied 11-norm regularization of the temporal Fourier 
coefficients associated to each pixel's timecourse. The algorithm could be written as the following minimization problem for the timecourse at spatial location $(x, y)$ :

$$
\begin{aligned}
s(x, y)= & \arg \min _{s(x, y)} \sum_{t} \mid m_{t}(x, y)-A(x, y) \sin (\omega t+\varphi) \\
& -\left.s_{t}(x, y)\right|^{2}+\lambda \sum_{l}\left|\hat{s}_{l}(x, y)\right|
\end{aligned}
$$

where $m$ is the measured image data along time for a given voxel, $\hat{s}$ is the Fourier transform in the temporal direction of the signal $s$ to be recovered, and $\lambda$ is the threshold value. In the following, the threshold used was $\lambda=500$ a.u. The variational problem can then be solved by a two-step algorithm. First, the amplitude, frequency, and phase of the flickering term $A(x, y) \sin (\omega t+\phi)$ are estimated while keeping $s$ constant, which reverts to an ordinary least-squares solution (initial conditions were empirically defined and led to problem convergence for all cases tested in this study, $A(x, y)=\max \left\{m_{t}(x, y)\right\}, \omega=3.10$, and $\left.\phi=1\right)$. Second, the flickering term is removed from the measurements and the signal $s$ is recovered by soft thresholding (threshold value $\lambda$ the Fourier coefficients of $m_{t}(x, y)-A(x, y) \sin (\omega t+\phi)$. Moreover, we estimate the parameters $\omega$ and $\phi$ globally for the complete dataset (and thus highly overdetermined), whereas the amplitude $A(x, y)$, which describes the amplitude of the flickering artifact, is chosen to vary spatially accordingly to our observations. Details concerning the algorithm implementation are described in the Appendix.

\section{Denoising Evaluation}

In order to estimate the efficiency of our image-enhancement algorithm, we used the basic cine sequence with $T R=13.5 \mathrm{~ms}$ and two averages as the reference (since the minimal $T R$ was $13.5 \mathrm{~ms}$ to achieve a spatial resolution of $257 \mu \mathrm{m}$ ), and we set the $T R$ of our interleaved cine to $27 \mathrm{~ms}$, in order to retrieve a final $T R$ of $13.5 \mathrm{~ms}$ and compare it to the reference. We performed that experiment on $n=8$ animals, on one middle short axis slice of the heart. Evaluation of the flickering artifact was done by computing the energy $E$ of the temporal derivative of the data term $\left\|I-I_{\text {ref }}\right\|^{2}$, where $I$ is the interleaved cine and $I_{\text {ref }}$ is the reference cine.

\section{E. Ex Vivo Analysis}

After the MRI session, mice were sacrificed and heart was removed. The left and right atria were excluded from the heart and the two ventricles were weighed on a precision balance to provide a reference value. This operation was performed by the same operator for all mice to avoid any bias introduced by the dissection method.

\section{F. Image Analysis}

The combination of the two image series for interleaved cine as well as the temporal regularization was performed with MATLAB (R2008b, The Mathworks, Inc., Natick, MA). Measurement of left and right ventricles mass as well as EF were done using Osirix software (open source http://www.osirix- viewer.com/). For mass evaluation, epicardial and endocardial contours of the left and right cavity were manually drawn on the short axis interleaved cine, in systole. The mass was calculated as follows:

$$
\begin{aligned}
(L V+R V)_{\mathrm{mass}}= & \gamma \cdot \text { Slice thickness } \cdot \sum_{\text {all slices }}[\text { epi area } \\
& -(L V \text { endo area }+R V \text { endo area })]
\end{aligned}
$$

where $\gamma$ is the specific gravity of the myocardium, $\gamma=$ $1.055 \mathrm{~g} / \mathrm{cm}^{3}$. Bland-Altman plot was used to compare the mass measured with MRI and the gold standard method (ex vivo weighed mass). The bias of the measure was given by the mean of the difference between MRI and ex vivo measurements.

For left ventricular EF (LVEF), endocardial contours of left ventricle were manually drawn on all slices, excluding the papillary muscles [14]. LVEF was defined as (LVEDVLVESV)/LVEDV, where LVEDV and LVESV are the left ventricular end-diastolic and end-systolic volumes. LV volumes as well as LVEF were measured on the interleaved cine as well as on the basic cine. For these measurements, basic cine was taken as the first repetition of the interleaved cine.

\section{G. Statistical Analysis}

Comparisons between groups were performed with a Student's $t$-test and, when appropriated, with a paired $t$-test. $P<0.05$ was considered as statistically significant.

\section{RESULTS}

An example of images obtained with the interleaved cine sequence was shown in Fig. 3. As observed in the temporal profile of the data [see Fig. 3(c)], the signal varied from one frame to the other resulting in a flickering effect when playing the cine movie. Examples of cine movies can be found at http: //miplab.unige.ch/pub/delattre1102p.php. This effect was not constant over the whole series; in this example, it was more important for the first phases of the cine and then reduced. Nevertheless, the proposed algorithm was efficiently denoising the image series by removing the flickering artifact.

\section{A. Quantitative Denoising Evaluation}

We evaluated the efficiency of our image-enhancement algorithm by comparing the interleaved cine with an acquired/reconstructed $T R$ of $27 / 13.5 \mathrm{~ms}$, respectively, to the reference cine ( $T R=13.5 \mathrm{~ms})$ as described in the method's section. Fig. 4 illustrates an example of the temporal profiles for the reference sequence and the interleaved cine before and after denoising. We observed as shown in this example a large reduction of the flickering artifact represented by the data term and its derivative $E$. The energy $E$ was significantly reduced after the denoising of interleaved cine images $\left(2.11 \pm 2.88 \times 10^{24}\right.$ a.u. before and $1.24 \pm 1.58 \times 10^{23}$ a.u. after denoising, respectively, $p=0.039)$ as a result of the decreased flickering artifact. Indeed, it corresponded to a noise reduction of $-10.4 \pm 3.8 \mathrm{~dB}$ $(n=8)$. 
(a)

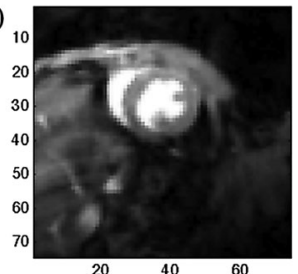

(b)
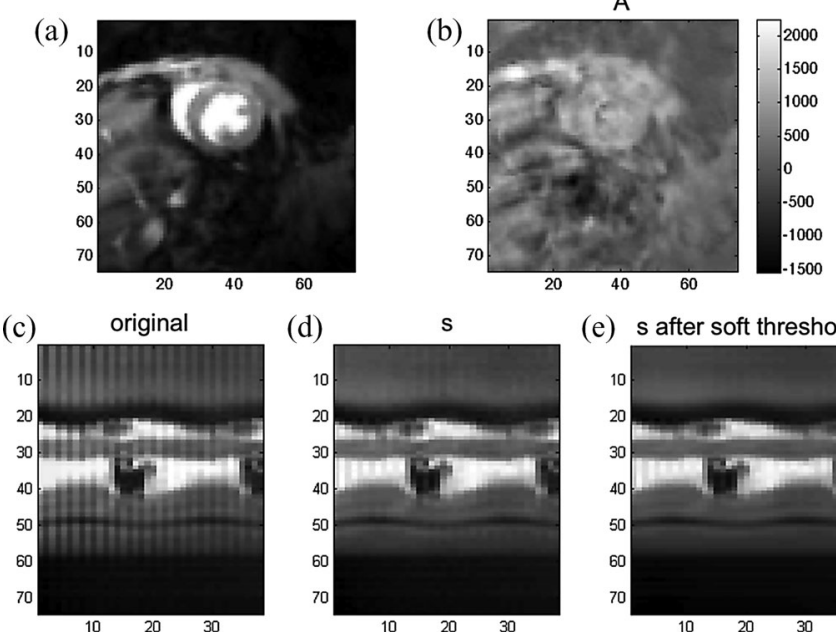

S

(d)

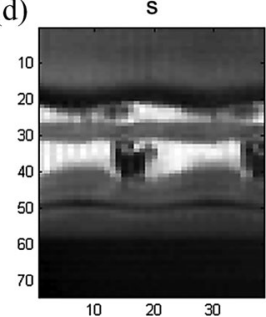

(e) s after soft threshold

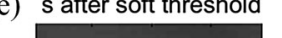

(a)

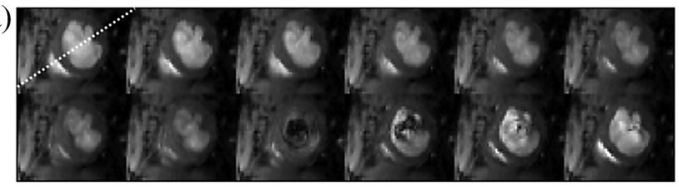

(b)

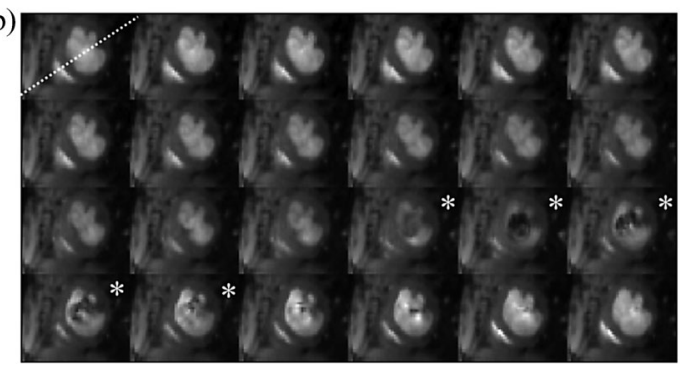

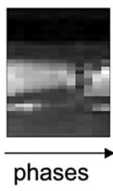

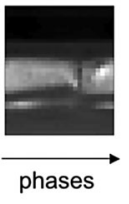

Fig. 3. (a) Image example of the interleaved cine series. (b) Parameter map $A$. The original temporal profile is very prone to flickering artifact (c), whereas the denoising algorithm removes it efficiently: (d) temporal profile of $s$ obtained after removing the component $A \sin (\omega k+\phi)$ of the data $f$, (e) final result of $s$ obtained after soft thresholding the Fourier coefficients $(\lambda=500$ a.u. $)$.

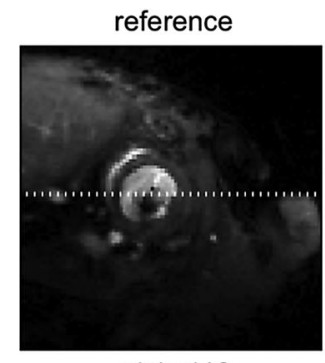

original IC
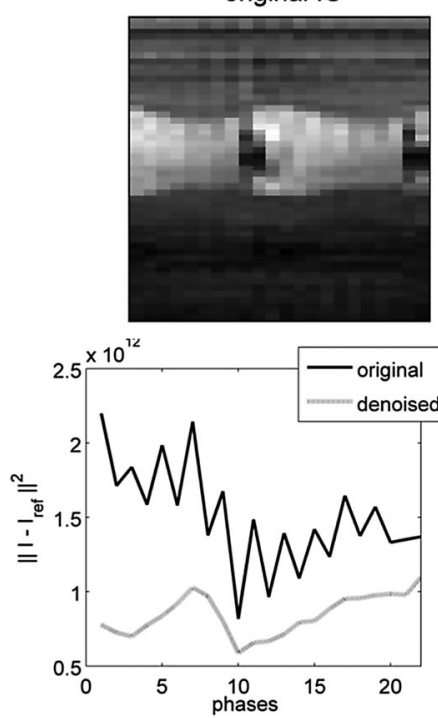

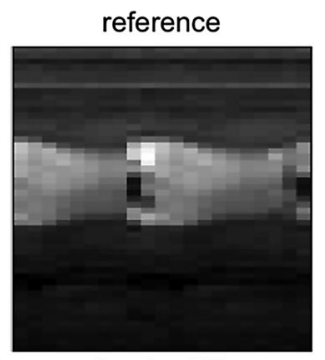

denoised IC
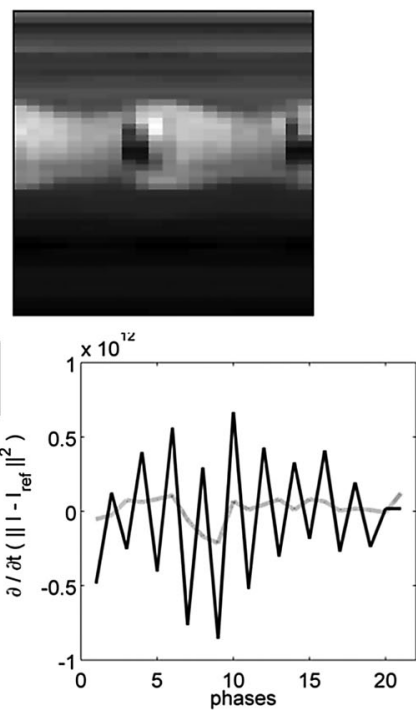

Fig. 4. (Upper row) Image example of the reference cine (TR $13.5 \mathrm{~ms}$, two averages, images are taken on two R-R cycles), temporal profile (taken on the white pixel line) for the reference cine as well as original profile for the interleaved cine (IC) (TR $27 \mathrm{~ms}$, no average, two repetitions). (Middle row) Temporal profile for original and denoised images. (Lower row) left: Effect of the denoising on the data term $\left\|\mathrm{I}-\mathrm{I}_{\mathrm{ref}}\right\|^{2}$; right: effect of denoising on the temporal derivative of the data term.

\section{B. Mass and Function Measurements}

To validate the interleaved cine sequence, we acquired short axis images of mice heart with a high temporal resolution; i.e., $T R=6.8 \mathrm{~ms}$. The increase in temporal resolution with the interleaved cine compared to the basic sequence is depicted in Fig. 5. We used the interleaved cine to measure the left and right ventricle masses of a group of control mice (8-12 weeks WT, $n=$ 4,23 weeks WT, $n=9$ ) and a group of mice that tend to develop a cardiac hypertrophy ( 23 weeks TG, $n=14$ ), total $n=27$. Fig. 6 shows an example of images of a WT and a highly hypertrophied TG mouse heart as well as the correlation between the two mass measurement methods. Mass measured with interleaved cine was highly correlated with ex vivo measurement $(y=0.829 x$ $\left.+25.1, R^{2}=0.858, p=4.15 \times 10^{-12}\right)$ and we observed no significant bias between methods (mean of the differences between MRI and ex vivo measurements was $-2.62 \mathrm{mg}$ ). When omitting the group presenting a cardiac hypertrophy, the masses were also highly correlated $\left(n=13, y=0.878 x+20.0, R^{2}=\right.$ $0.797, p=4.02 \times 10^{-5}$, bias was $3.34 \mathrm{mg}$ ).

Moreover, we measured the LVEF of a group of control mice (8-12 weeks WT, $n=4)$ and a group of mice with an infarction (8-12 weeks MI, $n=3$ ). As illustrated in Fig. 7, interleaved cine depicted accurately the contraction deficit present in the anterior region of the MI mice and in agreement with previous experiments [9]. We measured a significant increase of LVESV and LVEDV for MI group ( $p<0.01$ and $p<0.05$, respectively) as well as a significant decrease of the LVEF compared to the WT group $(p<0.001)$. In contrast, measurements using the basic cine sequence did not reveal a statistical difference of LVEDV between both groups. Moreover, the LVEF obtained with this sequence was significantly lower than the one obtained with interleaved cine $(p<0.05)$. Detailed values are presented in Table I. 


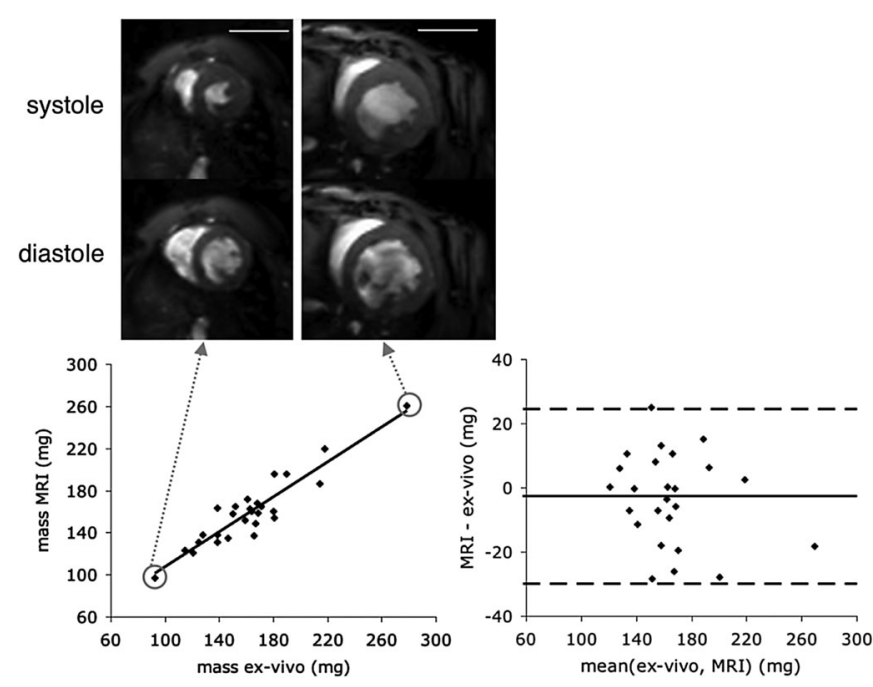

Fig. 6. (Left) Correlation of left and right ventricles mass measurement between MRI and with ex vivo methods, $y=0.829 x+25.1, R^{2}=0.858$. Images show examples of short axis systolic phases taken at a middle slice of the heart for a WT (left) and a TG mouse with hypertrophied myocardium (right) in systole (upper) and in diastole (bottom), white bar represents $5 \mathrm{~mm}$. (Right) Bland-Altman plot for comparison of MRI and ex vivo methods. Solid line is the mean of the differences between MRI and ex vivo methods $(-2.62 \mathrm{mg})$, dashed lines are $1.96 * \mathrm{SD}(\mathrm{MRI}$ ex vivo $)=27.15 \mathrm{mg}$ corresponding to $95 \%$ confidence interval.

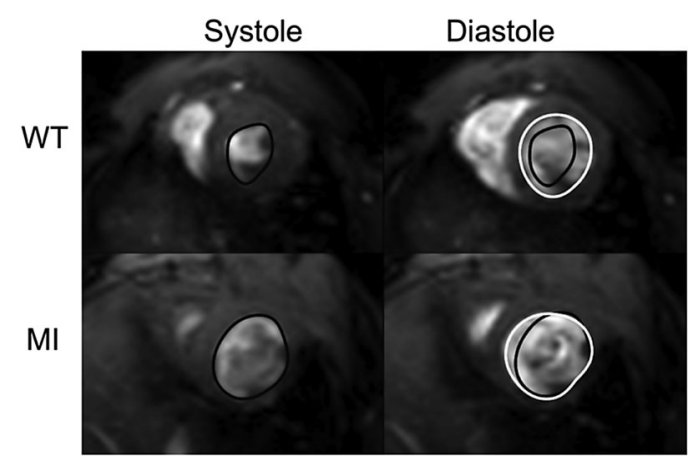

Fig. 7. Examples of middle slice of the heart for WT mouse (up) and MI mouse $24 \mathrm{~h}$ after surgery (down), in systolic and diastolic phases. Contours represent left ventricular cavity area for systole and diastole, lateral part of the myocardium exhibits no contraction for the MI mouse.

\section{DISCUSSION}

The evaluation experiment showed that the principle of combining two acquisitions shifted by half a $T R$ contributes to retrieving the temporal information of each cardiac phase. Indeed, by simply shifting the acquisition of the center of $k$-space (where the main part of the signal energy is located), we can choose the achieved temporal resolution. Consequently, the temporal resolution of the acquisition could be enhanced as high as the number of repetitions performed. However, there is little physiological interest to further enhance the number of repetitions for our application. Moreover, we believe that our method does add new information compared to a straight interpolation between cardiac phases because the duration of analog-to-digital converter sampling $(5 \mathrm{~ms})$ in our sequence is short compared to the $T R$ (mainly due to the time needed for slice selection). As an illustration, images acquired during the apparition of flow
TABLE I

VALUES OF LVESV, LVEDV, LVEF, AND HR FOR WT $(n=4)$ AND MI $(n=3)$ Mice Evaluated With InTERLEAVEd Cine AND Basic Cine

\begin{tabular}{cccc}
\hline Interleaved cine & WT & MI & \\
LVESV $(\mu \mathrm{l})$ & $25.6 \pm 3.4$ & $49.2 \pm 1.7$ & $\mathrm{p}<0.01$ \\
LVEDV $(\mu \mathrm{l})$ & $51.0 \pm 5.2$ & $66.2 \pm 1.6$ & $\mathrm{p}<0.05$ \\
LVEF $(\%)$ & $50.1 \pm 2.0$ & $25.7 \pm 1.2$ & $\mathrm{p}<0.001$ \\
Basic cine & WT & MI & \\
LVESV $(\mu \mathrm{l})$ & $26.2 \pm 3.3$ & $49.2 \pm 1.7$ & $\mathrm{p}<0.01$ \\
LVEDV $(\mu \mathrm{l})$ & $50.5 \pm 5.3$ & $62.6 \pm 2.7$ & $\mathrm{~N} . \mathrm{S}$. \\
LVEF $(\%)$ & $48.3 \pm 2.8$ & $21.4 \pm 1.5 *$ & $\mathrm{p}<0.001$ \\
HR $($ bpm $)$ & $503 \pm 18$ & $426 \pm 71$ & $\mathrm{~N} . S$ \\
\hline
\end{tabular}

$p$-values indicate significant differences between WT and MI group, $*$ indicates significant difference between the Two sequences $(p<0.05)$. Values are mean \pm SEM.
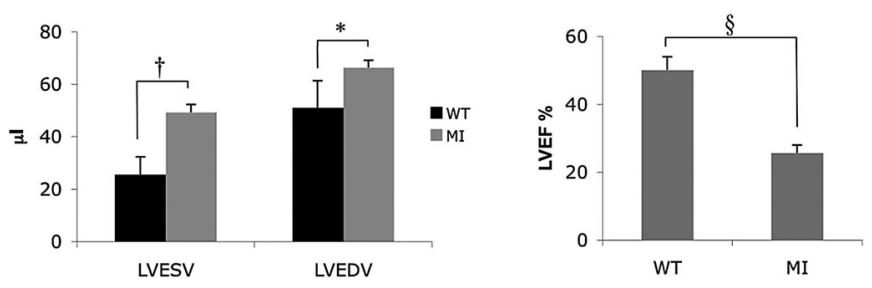

Fig. 8. LVESV, LVEDV, and LVEF for control mice, WT $(n=4)$, and for mice with an $\mathrm{MI}(n=3)$; values are mean $\pm \mathrm{SD}$, and symbols represent significant difference $\left({ }^{*} p<0.05,{ }^{\S} p<0.01,{ }^{\dagger} p<0.001\right)$.

artifacts in the blood pool cavity exhibit very different patterns from one image to the other [see images marked with an asterisk in Fig. 5(b)], that could not be retrieved with a simple linear interpolation.

The model used to reduce the flickering artifact was shown to be effective. The choice made to consider the parameter $A(x, y)$ as spatially dependent provided a map of the intensity of the flickering effect over the image stack. We observed in Fig. 3(b) that $A(x, y)$ presented some areas with negative values, especially located in parts of the image that were very corrupted by the flow artifacts and also around the descending aorta in which the flow is reversed compared to the heart. The sign of $A(x, y)$ may therefore give additional information concerning flow; however, this would require further investigations to be confirmed. Moreover, imposing sparsity pursuing regularization on the temporal Fourier coefficients using the 11-norm has also been used in recently proposed techniques such as compressed sensing [15], which have proven to be very efficient to solve the image-reconstruction inverse problem with a highly undersampled $k$ - $t$ space. However, in contrast to compressed sensing techniques, we do not use random sampling, neither $k-t$ undersampling. In our case, the limit is given by the sequence's $T R$, and not by the acquisition time. The denoising algorithm here is not used to recover missing data but to remove the artifact arising from the combination of two fully sampled image series acquired at different time points.

The threshold value $\lambda$ was set empirically by visual inspection to reach a compromise between reduction of the flickering artifact and keeping high contrast of heart motion and was kept the same for all experiments. A refinement of our method would be to find automatically the optimal threshold value. The difficulty in doing so is to define an objective criterion that would properly balance between noise reduction and conservation of 
temporal information. This was beyond the scope of this study but can be considered for future research.

High spatial and temporal resolutions are necessary to accurately estimate cardiac function and mass for small animal studies. However, MRI is a robust technique that can suffer from limited gradient performance, in particular on clinical scanners. The interleaved cine sequence proposed in this study achieved spatial and temporal resolutions comparable to those obtained on dedicated small animal scanners (i.e., $T R=6.8 \mathrm{~ms} /$ in-plane resolution $=257 \mu \mathrm{m} /$ slice thickness $=1 \mathrm{~mm}$ compared to $T R=$ $7.5 \mathrm{~ms} /$ in-plane resolution $=117 \mu \mathrm{m} /$ slice thickness $=1 \mathrm{~mm}$ for a $11.7 \mathrm{~T}$ scanner [16], or $T R=7 \mathrm{~ms} /$ in-plane resolution $=$ $100 \mu \mathrm{m} /$ slice thickness $=0.5 \mathrm{~mm}$ for a $9.4 \mathrm{~T}$ scanner [17]). To our knowledge, no previously published study performed on clinical scanner allowed to reach such high temporal and spatial resolutions without using dedicated gradient hardware. Despite the apparently high difference between our achieved spatial resolution and the one obtained on dedicated scanner, we have recently shown that in-plane resolution of $344 \mu \mathrm{m}$ was sufficient to observe the effect of pharmacological drug on the function improvement in a mice model of infarction [13]. In this study, it was possible to measure realistic mass, differences, highly correlated with ex vivo measurements, in a reasonable acquisition time. Moreover, the dispersion of data obtained by Bland-Altmann plot was higher than the values reported by Yang et al. [18] measured with a dedicated scanner but in the same range and corresponding to $17 \%$ variation of the mean mass value (95\% of data were located in the range of $\pm 27 \mathrm{mg}$ and $\pm 15 \mathrm{mg}$, for our study and Yang et al., respectively [18]). Our sequence could also efficiently depict the decrease of global function parameters between MI mice and WT group while this was not the case for all parameters with the basic cine. Moreover, the LVEF obtained with basic cine was significantly lower than the one obtained with interleaved cine due to an underestimation of LVEDV for the MI group. The values obtained for the WT group were indeed in good agreement with values reported by Ross et al. [19] for the same mice breed and age (they had $48 \mu 1,22 \mu 1$, and 50\% for LVEDV, LVESV, and LVEF, respectively).

\section{A. Future Applications}

This sequence can be advantageous for several applications, either in small animal or even in clinics. Indeed, this sequence renders possible the investigation of cardiac stress in a mouse model on clinical scanners. Moreover, and more generally, sequences needing a long preparation such as tagging can benefit from the interleaved cine framework to enhance the achievable temporal resolution [20], [21]. In clinics, highly time-resolved cine can be obtained in patients with this technique and may benefit to the observation of specific phases of the cardiac cycle such as isovolumetric contraction for measurement of moderateto-severe heart failure [22].

\section{B. Study Limitations}

The main limitation of the study is the absence of reference images allowing for direct validation of the highly time-resolved interleaved cine $(T R=6.8 \mathrm{~ms})$. Echography is not recognized as a gold standard in mice and we did not have access to a dedicated scanner to compare our measurements with those that could be obtained directly on systems with higher gradient performances. However, it would have been very difficult to reproduce the exact same physiological conditions between the two different imaging sessions, providing inherent sources of errors. In particular, the effect of anesthesia has been shown responsible for important variations in LVEDV and LVESV evaluation [23]. Finally, only indirect validation is proposed here through mass and function measurements, as it was previously proposed in several studies [8], [24]. Future work could induce pharmacological stress in mice to observe the impact on physiological parameters, such as LVEDV, LVESV, and function evaluation and verify the ability of our sequence to monitor cardiac function in stress conditions.

\section{CONCLUSION}

We proposed a sequence that doubles the temporal resolution initially available with the basic cine sequence on a clinical scanner. The interleaved cine is able to provide images with spatial and temporal resolutions compatible with the small animal imaging requirements without the addition of specific hardware; i.e., in-plane spatial resolution $257 \mu \mathrm{m}$ and repetition time $6.8 \mathrm{~ms}$, respectively. Therefore, this tool is essential for in-vivo assessment of cardiac function in mice and allows longitudinal studies to be performed on clinical scanners, more widely available than dedicated scanners.

\section{SUPPLEMENTARY MATERIAL}

Examples of cine movies for a mouse with an infarction $24 \mathrm{~h}$ after surgery can be found at http://miplab.unige.ch/pub/ delattre1102.php. Basic cine sequence as well as interleaved cine before and after the application of our image-enhancement algorithm are shown.

\section{APPENDIX}

\section{Description of the Algorithm}

Based on the observation that the flickering artifact was mainly due to different flow artifacts - the combined cine series was not necessarily in phase with the repetitions-we define the image model as the artifact-free signal degraded by additive terms for flickering and noise. Moreover, we could take advantage of the periodicity of the heart cycle to denoise the cine data by soft thresholding the (temporal) Fourier coefficients of the artifact-free signal. Therefore, the goal is to minimize the following functional for the timecourse $s$ at each spatial position $(x, y)$ :

$$
\begin{aligned}
s(x, y)= & \underset{s(x, y)}{\arg \min } \sum_{t} \mid m_{t}(x, y)-A(x, y) \sin (\omega t+\phi) \\
& -\left.s_{t}(x, y)\right|^{2}+\lambda \sum_{l}\left|\hat{s}_{l}(x, y)\right|
\end{aligned}
$$


where $k$ is the temporal index, $m$ is the measured data, $s$ is the denoised data with $\hat{\imath}$ its temporal Fourier transform. The algorithm was implemented as follows.

1) Estimation of data variation, calculation of spatial mean signal of $m_{k} \mu_{t}=1 / N_{x} \sum_{x} 1 / N_{y} \sum_{y} m_{t}(x, y)$, where $N_{x}$ and $N_{y}$ are the spatial dimensions $x$ and $y$ of measurement matrix $m_{k}$.

2) Fit of $\omega$ and $\phi$ for $\mu_{t}$ with a nonlinear least-squares optimization: $(\omega, \phi)=\arg \min \left|\mu_{t}-k \sin (\omega t+\phi)\right|^{2}$.

3) For each timecourse $m(x, y)$, fit $A(x, y)$.

4) Calculation of $s_{t}(x, y)=m_{t}(x, y)-A(x, y) \sin (\omega t+\phi)$.

5) Soft thresholding (shrinkage) of the temporal Fourier coefficients of $s(x, y)$.

The two first steps of the algorithm consisted in calculating the mean signal intensity variation of the image in function of time in order to evaluate $\omega$ and $\phi$ by a fit of the data. Indeed, we made the hypothesis that $\omega$ and $\phi$ are constant for all image pixels, whereas $A(x, y)$ can be spatially dependent. The third step of the algorithm is the estimation of the flickering amplitude $A(x, y)$. Knowing $\omega$ and $\phi$, it becomes a simple linear problem ( $s$ is considered null as an initial condition):

$$
\begin{aligned}
\frac{\partial J}{\partial A(x, y)}= & 0 \rightarrow-2 \sum_{t}\left(m_{t}(x, y)\right. \\
& -A(x, y) \sin (\omega t+\phi)) \cdot \sin (\omega t+\phi)=0 \\
A(x, y)= & \frac{\sum_{t} m_{t}(x, y) \sin (\omega t+\phi)}{\sum_{t} \sin ^{2}(\omega t+\phi)} .
\end{aligned}
$$

Knowing all the parameters of the model, the data $s$ are calculated with the relationship $s_{t}(x, y)=m_{t}(x, y)-$ $A(x, y) \sin (\omega t+\phi)$. The flickering artifact is therefore removed and the last step of the algorithm further reduces the residual noise in the image.

\section{REFERENCES}

[1] H. Ramakrishna, N. Feinglass, and J. G. Augoustides, "Clinical update in cardiac imaging including echocardiography," J. Cardiothorac. Vasc. Anesth., vol. 24, no. 2, pp. 371-378, Apr. 2010.

[2] W. G. Hundley, D. A. Bluemke, J. P. Finn, S. D. Flamm, M. A. Fogel, M. G. Friedrich, V. B. Ho, M. Jerosch-Herold, C. M. Kramer, W. J. Manning, M. Patel, G. M. Pohost, A. E. Stillman, R. D. White, and P. K. Woodard, "ACCF/ACR/AHA/NASCI/SCMR 2010 expert consensus document on cardiovascular magnetic resonance: A report of the American College of Cardiology Foundation Task Force on Expert Consensus Documents," Circulation, vol. 121, no. 22, pp. 2462-2508, Jun. 2010.

[3] G. W. Vick, III, "The gold standard for noninvasive imaging in coronary heart disease: Magnetic resonance imaging," Curr. Opin.Cardiol, vol. 24, no. 6, pp. 567-579, Nov. 2009.

[4] World Health Organization. (2011). "Cardiovascular diseases (CVDs)," vol. Fac sheet 317. [Online]. Available: http://www.who.int/mediacentre/ factsheets/fs317/en/index.html

[5] F. H. Epstein, "MR in mouse models of cardiac disease," NMR Biomed., vol. 20, no. 3, pp. 238-255, May 2007.

[6] W. D. Gilson and D. L. Kraitchman, "Cardiac magnetic resonance imaging in small rodents using clinical 1.5T and 3.0T scanners," Methods, vol. 43, no. 1, pp. 35-45, Sep. 2007.

[7] A. Roussakis, P. Baras, I. Seimenis, J. Andreou, and P. G. Danias, "Relationship of number of phases per cardiac cycle and accuracy of measurement of left ventricular volumes, ejection fraction, and mass," $J$. Cardiovasc. Magn. Reson., vol. 6, no. 4, pp. 837-844, 2004.

[8] F. Wiesmann, J. Ruff, S. Engelhardt, L. Hein, C. Dienesch, A. Leupold, R. Illinger, A. Frydrychowicz, K. H. Hiller, E. Rommel, A. Haase, M. J. Lohse, and S. Neubauer, "Dobutamine-stress magnetic resonance microimaging in mice: Acute changes of cardiac geometry and function in normal and failing murine hearts," Circ. Res., vol. 88, no. 6, pp. 563-569, Mar. 30, 2001.

[9] B. M. Delattre, V. Braunersreuther, J. N. Hyacinthe, L. A. Crowe, F. Mach, and J. P. Vallee, "Myocardial infarction quantification with manganeseenhanced MRI (MEMRI) in mice using a 3T clinical scanner," $N M R$ Biomed., vol. 23, no. 5, pp. 503-513, Jun. 2010.

[10] M. Makowski, C. Jansen, I. Webb, A. Chiribiri, E. Nagel, R. Botnar S. Kozerke, and S. Plein, "First-pass contrast-enhanced myocardial perfusion MRI in mice on a 3-T clinical MR scanner," Magn. Reson. Med., vol. 64, no. 6, pp. 1592-1598, Dec. 2010.

[11] A. A. Domenighetti, Q. Wang, M. Egger, S. M. Richards, T. Pedrazzini, and L. M. Delbridge, "Angiotensin II-mediated phenotypic cardiomyocyte remodeling leads to age-dependent cardiac dysfunction and failure," $\mathrm{Hy}$ pertension, vol. 46, no. 2, pp. 426-432, Aug. 2005.

[12] C. Pellieux, E. Aasum, T. S. Larsen, C. Montessuit, I. Papageorgiou, T. Pedrazzini, and R. Lerch, "Overexpression of angiotensinogen in the myocardium induces downregulation of the fatty acid oxidation pathway," J. Mol. Cell Cardiol., vol. 41, no. 3, pp. 459-466, Sep. 2006.

[13] F. Montecucco, V. Braunersreuther, S. Lenglet, B. M. Delattre, G. Pelli, V. Buatois, F. Guilhot, N. Vuilleumier, W. Ferlin, N. Fischer, J. P. Vallee, M. Kosco-Vilbois, and F. Mach, "CC chemokine CCL5 plays a central role impacting infarct size and post-infarction heart failure in mice," Eur. Heart J., published (May 23, 2011). [Online].

[14] R. J. vander Geest, V. G. M. Buller, E. Jansen, H. J. Lamb, L. H. B. Baur, E. E. vander Wall, A. de Roos, and J. H. C. Reiber, "Comparison between manual and semiautomated analysis of left ventricular volume parameters from short-axis MR images," J. Comput. Assisted Tomogr., vol. 21, no. 5, pp. 756-765, Sep./Oct. 1997.

[15] U. Gamper, P. Boesiger, and S. Kozerke, "Compressed sensing in dynamic MRI," Magn. Reson. Med., vol. 59, no. 2, pp. 365-373, Feb. 2008.

[16] N. Ojha, S. Roy, J. Radtke, O. Simonetti, S. Gnyawali, J. L. Zweier, P. Kuppusamy, and C. K. Sen, "Characterization of the structural and functional changes in the myocardium following focal ischemia-reperfusion injury," Am. J. Physiol. Heart Circ. Physiol., vol. 294, no. 6, pp. H2435H2443, Jun. 2008.

[17] E. M. Winter, R. W. Grauss, D. E. Atsma, B. Hogers, R. E. Poelmann, R. J. van der Geest, C. Tschope, M. J. Schalij, A. C. Gittenberger-de Groot, and P. Steendijk, "Left ventricular function in the post-infarct failing mouse heart by magnetic resonance imaging and conductance catheter: A comparative analysis," Acta Physiol. (Oxf.), vol. 194, no. 2, pp. 111122, Oct. 2008.

[18] Z. Yang, S. S. Berr, W. D. Gilson, M. C. Toufektsian, and B. A. French, "Simultaneous evaluation of infarct size and cardiac function in intact mice by contrast-enhanced cardiac magnetic resonance imaging reveals contractile dysfunction in noninfarcted regions early after myocardial infarction," Circulation, vol. 109, no. 9, pp. 1161-1167, Mar. 2004.

[19] A. J. Ross, Z. Yang, S. S. Berr, W. D. Gilson, W. C. Petersen, J. N. Oshinski, and B. A. French, "Serial MRI evaluation of cardiac structure and function in mice after reperfused myocardial infarction," Magn. Reson. Med., vol. 47, no. 6, pp. 1158-1168, Jun. 2002.

[20] J. L. Daire, J. P. Jacob, J. N. Hyacinthe, P. Croisille, K. Montet-Abou, S. Richter, D. Botsikas, M. Lepetit-Coiffe, D. Morel, and J. P. Vallee, "Cine and tagged cardiovascular magnetic resonance imaging in normal rat at 1.5T: A rest and stress study," J. Cardiovasc. Magn. Reson., vol. 10, p. $48,2008$.

[21] J. N. Hyacinthe, M. K. Ivancevic, J. L. Daire, and J. P. Vallee, "Feasibility of complementary spatial modulation of magnetization tagging in the rat heart after manganese injection," NMR Biomed., vol. 21, no. 1, pp. 15-21, Jan. 2008 .

[22] H. V. Dinh, J. Alvergue, J. Sayre, J. S. Child, V. S. Deshpande, G. Laub, and J. P. Finn, "Isovolumic cardiac contraction on high-temporal-resolution cine MR images: Study in heart failure patients and healthy volunteers," Radiology, vol. 248, no. 2, pp. 458-465, Aug. 2008.

[23] F. Kober, I. Iltis, P. J. Cozzone, and M. Bernard, "Cine-MRI assessment of cardiac function in mice anesthetized with ketamine/xylazine and isoflurane," MAGMA, vol. 17, no. 3-6, pp. 157-161, Dec. 2004.

[24] S. E. Slawson, B. B. Roman, D. S. Williams, and A. P. Koretsky, "Cardiac MRI of the normal and hypertrophied mouse heart," Magn. Reson. Med., vol. 39, no. 6, pp. 980-987, Jun. 1998.

Authors' photographs and biographies not available at the time of publication. 